\title{
Intelligente Roboter in Interaktion mit Menschen
}

\author{
H. Malleck OVE, C. Mecklenbräuker
}

Online publiziert am 11. November 2019

(c) Springer-Verlag GmbH Austria, ein Teil von Springer Nature 2019

"Jede Innovation (Wahrheit) tritt zuerst als Phantasterei (Irrlehre) in die Welt, denn die Welt ist immer von gestern." Frei nach Egon Friedell

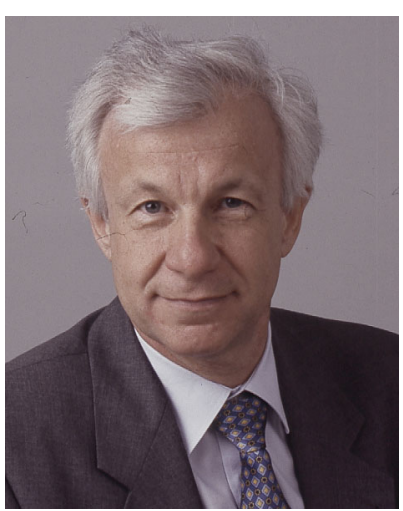

Helmut Malleck

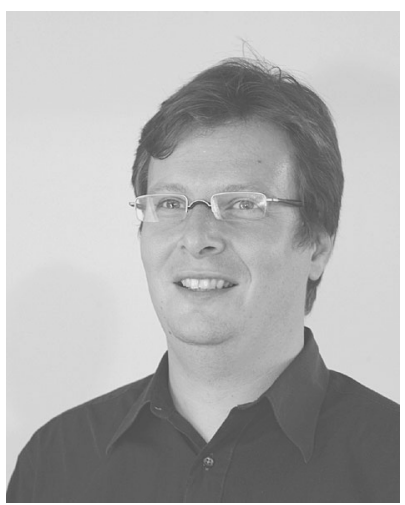

Christoph Mecklenbräuker Leser,
Liebe Leserin und lieber

das Thema „Intelligente Roboter in Interaktion mit Menschen" aufzugreifen und für Sie das vorliegende e\&i-Themenheft zusammenzustellen, faszinierte uns; kommen doch die allermeisten von uns tagtäglich mit Automaten, manche auch mit Robotern, in Berührung. Beinahe täglich finden wir in der Tagespresse Meldungen über künstliche Intelligenz (artificial intelligence $\mathrm{Al}$ ), wie beispielsweise über "WienBot", den digitalen Assistenten der Stadt Wien, oder über "Ai-Da", die als erste ultrarealistische humanoide Al-Roboterkünstlerin gesehen wird. Drohnen möglicherweise bald autonom - absolvierten Jungfernflüge als Flugtaxis. Autonome Drohnen überprüfen nicht nur Lack- und Strukturschäden der Flugzeuge. Menschengroße Roboter, die mit künstlicher Haut und 10.000en Sensoren ihre Umgebung erspüren - der " $\mathrm{H}-1$ - -, werden erforscht. Zukunftsromane projizieren den partnerschaftlichen Umgang von Menschen und Robotern ${ }^{1}$. Von Neuerscheinungen am Büchermarkt wird auch hinterfragt, ob Zukunftstechnologien endlos langes menschliches Leben ermöglichen könnten ${ }^{2}$. Intelligente Roboter in Interaktion mit Menschen: mehr als nur eine Vision? Die Zukunft hat längst begonnen ..

Gerne nehmen wir die Dienste „dummer" Roboter, beispielsweise zum Staubsaugen oder zum Rasenmähen, in Anspruch. In der industriellen Fertigung werden seit Jahren für Menschen schwer leistba-

${ }^{1}$ McEwan, Ian „Maschinen und ich", Diogenes Verlag, Zürich 2019.

2Beigbeder, Frédéric und Schoch, Julia „Endlos Leben”, Piper Verlag GmbH, München 2018. re oder gleichförmige Arbeiten an, durch Schutzzäune eingegrenzte, Roboter delegiert. In Labors wird jedoch an drahtlos operierenden (humanoiden) Robotern, an autarkem und autonomem Fahren - autonomes Fahren von Robotertaxis ist mancherorts schon Realität - und an der Steuerung von Drohnen gearbeitet. In absehbarer Zeit werden hohe 5G-Netzkapazitäten die Fernsteuerung der intelligenten Roboter ermöglichen. Darüber hinaus werden die Entwicklungen im Bereich der künstlichen Intelligenz den Einsatz autonomer Roboter in partnerschaftlichem Miteinander mit Menschen beschleunigen.

Gewiss, für das partnerschaftliche Interagieren von autonomen Robotern und Menschen, für ein physisches Miteinander, ist noch viel Forschungs- und Entwicklungsarbeit zu leisten. Man ist immer noch weit davon entfernt, dass Menschen und Roboter kollaborativ am selben Werkstück zusammenarbeiten. Dabei ist einerseits die Frage der Mitarbeitersicherheit, andererseits sind automatisierte Objekterfassung und cyber-physische Robotersicherheit im Fokus. Das Angerempeltwerden des menschlichen Kollegen durch den autonomen Roboter, unelastische Stöße im kognitiven Prozess, sind dabei nur eines der Probleme. Könnte durch künstliche Intelligenz auch robotische Empathie möglich werden? Die Beiträge in diesem e\&iThemenheft geben keine Antwort auf die Frage, ob und wann Roboter mit Menschen auf Augenhöhe interagieren.

Ein offener Punkt ist auch, ob unsere Gesellschaft die Interaktion von Menschen mit intelligenten autonomen Robotern akzeptieren will. Sicher können intelligente autonome Roboter bei der Lösung aktuell anstehender globaler Herausforderungen nützen. Jedenfalls werden ökonomische Gesichtspunkte den zukünftigen Einsatz von intelligenten Robotern in Interaktion mit der Natur - mit Menschen, Tier und Vegetation - wesentlich beeinflussen.

Das vorliegende e\&i-Themenheft umfasst acht Originalarbeiten. Im einleitenden Beitrag wird die öffentliche Meinung gegenüber neuen autonomen Robotern erhoben. Die anschließenden Beiträge erörtern Fragen zur Robotersicherheit sowie zu kognitiver und zu sicherer kollaborativer Produktion. Recht unterschiedliche MenschRoboter-Kooperation bei der Programmierung von Industrierobotern stellen die folgenden zwei Beiträge dar. Den Abschluss bildet ein Beitrag über die Interaktion von Robotern mit Pflanzen zur Steuerung des Pflanzenwachstums.

\section{Originalarbeiten zum Themenschwerpunkt}

- Im Beitrag von Timo Gnambs „Attitudes towards Emergent Autonomous Robots in Austria and Germany" werden einleitend mögliche Einsatzbereiche für autonome Roboter angeführt und deren Akzeptanz in unserer Gesellschaft beleuchtet. Autonome

Malleck, Helmut, Arbeitskreis für technische Koordination für öffentliche Kommunikationsnetze und -dienste (AK-TK), Postfach 147, 1006 Wien, Österreich (E-Mail: ak-tk@oefeg.at); Mecklenbräuker, Christoph, Technische Universität Wien Institute of Telecommunications, Gußhausstraße 25-25a, 1040 Wien, Österreich 
Roboter sind unverzichtbar in zahlreichen Industriezweigen und stehen kurz davor, auch andere Anwendungsfelder wie Verkehr und Gesundheitsversorgung zu erobern. Die vorliegende Studie untersucht nun die öffentliche Meinung gegenüber neuen automatischen Systemen in Österreich und Deutschland. Die Ergebnisse lassen vermuten, dass sich bei zunehmender Erfahrung mit Robotern die negativen Seiten von Automation verdeutlichen und die Skepsis für deren verbreitete Nutzung steigt.

- Für eine Mensch-Roboter-Kollaboration gehen Michael Hofbaur und Michael Rathmair in ihrem Beitrag "Physische Sicherheit in der Mensch-Roboter-Kollaboration" von einer gesamtheitlichen Betrachtung der Roboteranwendung und des Arbeitsumfeldes aus. Dabei haben sowohl das Werkstück als auch die Bauart, der Greifer und der Bewegungsablauf des Roboters großen Einfluss auf die Sicherheitsbeurteilung. Für kollaborative Roboteranwendungen einzuhaltende Grenzwerte am menschlichen Körper werden diskutiert und deren messtechnische Validierung für ein zerstörungs- und verletzungsfreies Miteinander von Menschen und Roboter erörtert. Diese Thematik wurde auch beim IT-Kolloquium 2019 präsentiert.

- Der Beitrag von Willibald Krenn „Sicherheit vernetzter, hochautomatisierter Roboter" thematisiert die Herausforderungen an operationelle Sicherheit (Safety) und Cyber-Sicherheit (Security) von hochautomatisierten und vernetzten Robotern. Für ein strukturiertes Monitoring zur Cyber-Sicherheit werden modellbasierte Analysemethoden, ebenso wie Standardisierungsvorgaben, beschrieben und auf die starke Überlappung von Cyber-Sicherheit mit künstlicher Intelligenz näher eingegangen. Der Beitrag ist eine erweiterte und aktualisierte Version des gleichlautenden Vortrags beim IT-Kolloquium 2019.

- Georg Weichhart et al. „Human/Machine/Roboter: Technologies for Cognitive Processes" gibt einen aktuellen Überblick zu den laufenden Forschungsaktivitäten in dem vor zwei Jahren gegründeten Forschungszentrum Pro ${ }^{2}$ Future. In Projekten von Pro ${ }^{2}$ Future werden kognitive Ansätze in der Produktion untersucht. Erste Forschungsergebnisse dazu zeigen neue Möglichkeiten in der Mensch-Roboter-Kommunikation bei der nahtlosen Rekonfiguration von robotischen Systemen. Diese erlauben detaillierte, statistische Einblicke in flexible Produktionssysteme. Der Beitrag geht auf einen Vortrag beim IT-Kolloquium 2019 zurück.

- Der Fokus des Beitrags von Vinzenz Sattinger et al. „Methodik zur Entwicklung sicherer kollaborativer Produktionssysteme im Rahmen von Industrie 4.0" richtet sich auf die Erstellung einer Roadmap zur Unterstützung von Unternehmen bei Entwicklung und Einführung kollaborativer Produktionssysteme. Ausgegangen wird dabei von dem für mechatronische Systeme bewährten VModell, das um Normen und Richtlinien zu Safety und IT-Security in allen Phasen des Konstruktionsprozesses erweitert wurde, sodass sich domänenübergreifende, sicherheitstechnisch zertifizierbare Gesamtlösungen ergeben. Im Beitrag wird die Methodik für einen mobilen Manipulator näher diskutiert, sie ist aber auch auf Verkettungen von mobilen Robotern und Industrierobotern anwendbar.

- Im Beitrag "Skill based Programming of Complex Robotic Assembly Tasks for Industrial Applications" von Sharath Chandra Akkaladevi et al. wird die Mensch-Roboter-Kooperation auf Basis
Programmierung der Industrieroboter zur Serienproduktion mit Losgröße eins erläutert. Dabei reduziert ein neues von Hand geführtes Prozesswerkzeug den Programmieraufwand. Darüber hinaus wird eine effiziente Lernmethode für die Aufgabenreihenfolge in Montageprozessen vorgestellt, bei welcher die erforderliche Prozessinformation über eine interaktive grafische Benutzeroberfläche mit künstlicher Intelligenz aufbereitet wird. Mit Beispielen werden die Benutzerfreundlichkeit der flexiblen Programmierwerkzeuge sowie das technologische Potenzial belegt.

- In ihrem Beitrag „Multilingual Speech Control for ROS-driven Robots" beschreiben Dominik Patrick Hofer und Felix Strohmeier eine automatische Sprachsteuerung mit Spracherkennung als wesentliches Element zur natürlichen Zusammenarbeit zwischen Menschen und Robotern (ROS... Robot Operating System). Um zukünftigen Anforderungen, beispielsweise neuen Roboterfähigkeiten, gerecht werden zu können wurde die Systemarchitektur erweiterbar gestaltet. Die modular aufgebaute Pilotimplementierung nutzt Cloud-Dienste und Open-Source-Implementierungen und wurde im Szenario mit einem kollaborativen Roboterarm einerseits und einem sich autonom bewegenden Roboterfahrzeug andererseits erfolgreich getestet.

- Dem neuen Ansatz zur Lebensmittelproduktion, bei welchem Pflanzen nicht mehr am Feld, sondern in Gebäuden vertikal gestapelt in Behältern, ohne Erde und Mineralien, angepflanzt sind, widmet sich der Beitrag von Clemens Gnauer et al. „Towards a secure and self-adapting smart indoor farming framework". Durch die Interaktion von Robotern mit den Pflanzen wird das Pflanzenwachstum in einer Luft- und Nährstoffumgebung gesteuert. Zu diesem Indoor Farming Support as a Service (IFSaaS) wird ein Prototyp vorgestellt, der aus mehreren loT-Komponenten und einem Sprühroboter (AgroRobot) besteht. Bei sich ändernden Umwelteinflüssen verarbeitet eine sichere, skalierbare und selbstadaptierende Robotersteuerung große Datenmengen für optimales Pflanzenwachstum unter Beachtung von Safety- und SecurityAspekten.

\section{Weiterführendes Thema als Bericht}

- Darüber, dass autonome Flug-Roboter auch bei uns Alltag werden könnten, berichtet der Beitrag „Autonome Luftfahrzeuge bald auch in Österreich, Erfolge einer Partnerschaft von FACC mit EHang" in der Rubrik Praxis + Wissen.

Der Traum des Menschen, intelligente Maschinen mit nahtloser Mensch-Roboter-Interaktion zu bauen, ist uralt und könnte bald Wirklichkeit werden. Spekulationen über Szenarien, in denen künstliche Intelligenz an menschliche Geisteskraft heranreicht, überlassen wir gerne Science-Fiction-Romanen. Künstliche Intelligenz und Robotik sind jedenfalls drauf und dran, unser Leben bald komplett zu verändern.

Eine spannende Lektüre der Beiträge dieses e\&i-Themenhefts und angeregte Diskussionen über intelligente Roboter in Interaktion mit Menschen wünschen Ihnen

Helmut Malleck und Christoph Mecklenbräuker

Hinweis des Verlags Der Verlag bleibt in Hinblick auf geografische Zuordnungen und Gebietsbezeichnungen in veröffentlichten Karten und Institutsadressen neutral. 